\title{
LACTANCIA MATERNA: IMPACTO DE LA CONSULTA DE APOYO A LA MADRE QUE TRABAJA
}

NOY, S. E.; APIP, P.M.; GUZMAN, P.P. Lactancia materna: impacto de la consulta de apoyo a la madre que trabaja. Rev.latino-am.enfermagem, Ribeirão Preto, v. 6, n. 3, p. 61-70, julho 1998.

La presente investigación, descriptiva exploratoria, retrospectiva y transversal, pretende responder el siguiente cuestionamiento: ¿ Cuál es el impacto que la Consulta de apoyo a la madre que trabaja, del Centro de Diagnóstico de la Pontificia Universidad Católica de Chile, tiene en la prolongación de la lactancia materna?. La población estudiada la constituyen 82 madres atendidas en la Consulta de apoyo, durante el período de marzo y septiembre de 1995, de las cuales se tomó una muestra de treinta madres. El impacto de esta consulta fue evaluado a través de un instrumento que se aplicó por entrevista dirigida a cada una de las madres. Los datos fueron analizados estadísticamente con el programa estadístico EPIINFO, el método de sobre vida de Kaplan-Meier y el test de Mantel-Haenszel para comparar curvas de sobre vida. Al analizar los datos se encontró que las madres estudiadas son en su mayoría adultas jóvenes, con pareja estable, primíparas, con un nivel de educación técnico y/o profesional y que se desempeñan mayoritariamente como empleadas de oficina. Tienen una jornada laboral completa, y existe una diferencia significativa entre el sueldo mínimo y el máximo que perciben. Los resultados de este estudio permiten concluir que las madres lograron una lactancia materna exclusiva y edad de destete ideal. El poder de resolución de la Consulta según las madres resultó satisfactorio. El factor reforzador más significativo en relación al aumento de la probabilidad de continuar amamantando, es el apoyo que recibe la madre después de su reincorporación laboral. Finalmente la Consulta tiene un buen impacto.

TÉRMINOS CLAVES: lactancia, madre trabajadora, factores influyentes, prolongación de la lactancia, destete

\section{INTRODUCCIÓN}

El número de madres que interrumpe o disminuye su lactancia debido a la reincorporación a su trabajo es cada vez mayor. En Chile en el año 1990 el porcentaje de niños que reciben lactancia materna exclusiva (L.M.E.) a los cuatro meses de edad corresponde a un $44.1 \%$ y el porcentaje de lactancia materna parcial a los doce meses de vida corresponde a un $19.7 \%$. La reinserción laboral de la mujer una vez finalizado el período de descanso postnatal, constituye una de las causas de mayor incidencia en la introducción precoz de alimentación complementaria al niño (SCHELLHORN \& VALDÉS, 1995), con todos los costos negativos que ello tiene tanto para él como para su madre. En Chile las disposiciones legales que protegen directamente la lactancia materna son: (MINSAL, 1993).

1. Descanso post-natal. Correspondiente a doce semanas después de la fecha de parto.

2. Licencia para el cuidado del niño menor de un año en caso de enfermedad grave.
3. Derecho a sala cuna (derechos a sala cuna para los niños menores de dos años).

4. Tiempo para alimentar (1 hora para alimentar al niño menor de dos años).

5. De acuerdo al artículo 192 del Código del Trabajo Chileno, corresponde a la Junta Nacional de Jardines Infantiles (JUNJI) y a la Dirección del Trabajo fiscalizar el cumplimiento de estas normas. Cualquier persona puede denunciar ante dichos organismos las infracciones de que tuviere conocimiento.

Al revisar detenidamente la protección legal contenida en el Código del Trabajo Chileno se debe considerar lo siguiente:

- El descanso post-natal es válido sólo para aquellas mujeres que tienen un contrato de trabajo y su previsión al día, quienes en Chile representan aproximadamente el $40 \%$ de la población femenina económicamente activa.

- Se ha constatado que las madres que no cuentan con este derecho, abandonan precozmente su lactancia para continuar trabajando y poder percibir sus ingresos

\footnotetext{
* Enfermera-Matrona, engresada de la Escuela de Enfermería de la Pontificia Universidad Católica de Chile

** Enfermera-Matrona, engresada de la Escuela de Enfermería de la Pontificia Universidad Católica de Chile

*** Enfermera-Matrona, magister en Salud Pública, profesor Adjunto. Jefe del Depto de Enfermería en salud mental y Psiquiatría Escuela de Enfermería Pontificia Universidad Católica de Chile
} 
económicos. Diversas investigaciones han demostrado la correlación negativa entre trabajo materno y la duración de la lactancia. Esta asociación adquiere mayor importancia a partir del tercer mes de vida del lactante, lo que refleja por una parte, el efecto protector que tiene el permiso post-natal en la lactancia materna, y por otra la falta de apoyo para que la madre que trabaja pueda continuar la lactancia una vez finalizado dicho período (CASADO et al., 1992).

- El derecho a sala cuna y tiempo para alimentar al hijo menor de dos años es obligatorio sólo para aquellos empleadores que cuentan con 20 o más mujeres trabajando. Aunque no se cuenta con cifras nacionales de cobertura de salas cuna se puede deducir que el porcentaje de mujeres que en el país tiene acceso a ellas es muy bajo. Sólo el 1.1\% de los niños menores de dos años, son atendidos por salas cunas pertenecientes a la JUNJI, a la Fundación Nacional de Desarrollo Integral del Menor (INTEGRA), y otros organismos. Este hecho dificulta aún más la posibilidad de las madres trabajadoras de continuar amamantando.

- Si se considera que la participación femenina en la población económicamente activa ha ido aumentando en forma sostenida durante los últimos 30 años, alcanzando la tasa de participación laboral de la mujer un $33.4 \%$ en 1992; y a esto se agrega que las mujeres alcanzan sus mayores cifras de actividad laboral hacia los 25 años, manteniéndose prácticamente estables hasta los 50 (COURT, 1996); se puede ver que es justamente la población femenina en edad reproductiva la que desempeña una actividad remunerada fuera del hogar.

GREEN et al.(1980) plantea en el modelo PRECEDE, un paradigma de planificación y evaluación educativa en salud, el que presenta pasos lógicos y sistemáticos que evitan las aproximaciones tradicionales en educación para la salud, incorporando en ésta un enfoque multidimensional y multifactorial. En él se consideran la evaluación de los factores que predisponen, facilitan o refuerzan conductas en salud sobre las cuales se trabajan programas educativos (GUZMAN et al., 1990). En este caso, los factores predisponentes son todos aquellos que están relacionados con la motivación de la madre hacia su lactancia; los factores facilitadores son aquellos que tienen relación con las habilidades y recursos necesarios para prolongar la lactancia; los factores reforzadores son todos los que significan un incentivo continuo para la madre y la apoyan en el mantenimiento de su lactancia. Para la mantención de la lactancia existen diversos factores involucrados que a veces no dependen sólo de la conducta del individuo, sino de aspectos externos que no se pueden cambiar: apoyos familiares, leyes sociales, reglamentos de trabajo entre otros (GALAZ et al., 1994).
En consecuencia el hecho que estas mujeres mantengan la lactancia, es una necesidad cada vez más importante y constituye el desafío actual de los profesionales de la salud interesados en la promoción de la lactancia materna (VALDÉS et al.,1994). Equipos de dichos profesionales con la motivación y los conocimientos necesarios, han creado instancias de ayuda como la Consulta de apoyo a la madre que trabaja en el Centro de Diagnóstico de la Pontificia Universidad Católica de Chile (C.E.D.I.U.C). Esta actividad, consiste en una consulta grupal dirigida por una enfermera matrona en la cual las madres comparten sus experiencias, se interiorizan con respecto a los beneficios legales que les corresponden, aprenden y practican la extracción manual de leche y reciben los conocimientos necesarios para la conservación, transporte, almacenamiento y administración de la leche extraída.

Con el fin de mejorar y optimizar los recursos destinados a dicho propósito, es necesario evaluar el impacto de esta modalidad de atención sobre los índices de lactancia materna de las madres asistidas en la consulta de apoyo.

El Objetivo General de la investigación es determinar el impacto que la consulta de apoyo tiene en la prolongación de la lactancia materna en las madres que asisten al Servicio de Pediatría del C.E.D.I.U.C.. Los Objetivos Específicos son:

1. Identificar los factores predisponentes, facilitadores y reforzadores que determinan el impacto de la Consulta de apoyo en la prolongación de la lactancia materna.

2. Determinar el tiempo de duración de la L.M.E. en el grupo de estudio, el destete e introducción de otros alimentos.

3. Identificar las principales causa de abandono de la lactancia materna.

4. Valorar la adherencia a los acuerdos tomados en la consulta.

5. Estimar el poder resolutivo de la consulta percibido por las madres.

6. Evaluar el cumplimiento de los derechos legales de la madre en este grupo de estudio.

\section{MATERIAL Y MÉTODO}

El presente estudio es retrospectivo, transversal de tipo descriptivo exploratorio.

El universo estudiado son las madres que fueron atendidas en la Consulta de apoyo a la madre que trabaja, en el servicio de pediatría del C.E.D.I.U.C, entre los meses de marzo y septiembre de 1995. Como criterio de inclusión para la unidad muestral, se consideró a todas las madres cuyos hijos tenían alrededor de un año de edad en la fecha de aplicación del instrumento. Esto con la 
finalidad de evaluar la edad ideal del destete. También en la ficha de atención de la consulta de apoyo de estas madres, debía aparecer consignado su número de teléfono. Se excluyó de la unidad muestral al resto de las madres por la dificultad para poder ubicarlas. El total de la muestra fué de treinta madres (35\%) de un universo de 82.

Este estudio se realizó en tres etapas:

a) Primera etapa.

Actividades realizadas:

- Aproximación y conocimiento de la Consulta de Apoyo por parte de las investigadoras.

- Revisión de Fichas de las madres que asisten a la Consulta de Apoyo e investigación bibliográfica sobre el tema.

- Evaluación pre-diagnóstica en la cual se identificaron factores presentes en las madres que influyen en la prolongación de la lactancia.

- En relación a los planteamientos del modelo PRECEDE se construyó un instrumento de evaluación de la consulta de apoyo, con preguntas destinadas a identificar la presencia de factores predisponentes, facilitadores y reforzadores en la mantención de la lactancia de estas madres (Cuadro 1). Fue validado a través de la revisión de jueces y de una aplicación piloto (Anexo 1).

Cuadro 1 - Factores predisponentes, facilitadores y reforzadores de la lactancia materna considerados en el estudio

\begin{tabular}{|l|l|}
\hline Factores predisponentes & $\begin{array}{l}\text { Mitos y creencias de la madre, } \\
\text { experiencias anteriores. }\end{array}$ \\
\hline Factores facilitadores & $\begin{array}{l}\text { Compromiso social existente con } \\
\text { la madre que trabaja (beneficios } \\
\text { legales y facilidades otorgadas). }\end{array}$ \\
\hline Factores reforzadores & $\begin{array}{l}\text { Apoyo de per sonas cerc anas, } \\
\text { relación entre la madre y los } \\
\text { profesionales de la salud. }\end{array}$ \\
\hline
\end{tabular}

b) Segunda etapa.

Actividades realizadas:

- Aplicación del instrumento de evaluación del impacto de la consulta de apoyo. Se realizó con la siguiente modalidad:

Contacto telefónico con la madre y acuerdo de reunión en lugar de trabajo para aplicar el instrumento.

c) Tercera etapa.

Actividades realizadas:

- Interpretación, tabulación y análisis de los datos. Los datos fueron analizados estadísticamente con el software EPIINFO, para determinar las diferencias significativas obtenidas, se utilizó el análisis de sobre vida de KaplanMeier.

EPIINFO es un software (conjunto de programas computacionales) que permite realizar los cálculos básicos y avanzados de la estadística, realizando a la vez algunos análisis específicos solicitados por el usuario tales como el Análisis de Kaplan-Meier, sobre los datos recogidos en una investigación científica. Este es un programa que pertenece e interactúa con la generación de software de DBASE, LOTUS 123, QPRO, etc. Fue desarrollado por OMS y C.D.C.

El análisis de sobre vida de Kaplan-Meier consiste en una comparación de probabilidades de dos variables frente a un fenómeno. A las curvas obtenidas del análisis de Kaplan-Meier fue posible aplicar el Test de MantelHaenzel ya que la muestra utilizada lo permitió arrojando un valor $\mathrm{p}=0.0182 \mathrm{el}$ cual equivale a un porcentaje mínimo de posibilidad de error en este resultado (si $\mathrm{p}<0.05$ el resultado es significativo).

Se definió el impacto, como el efecto que tiene la consulta de apoyo en la lactancia materna del grupo en estudio, el cual está determinado por 6 dimensiones cada una de las cuales fue medida por una subdimensión. A cada una de ellas le fué asignada un puntaje correspondiendo 1 al mínimo esperado. De acuerdo a la suma total de los puntajes obtenidos por el grupo en cada ítem, se obtuvo un puntaje total el cual se clasificó en alguna de las siguientes categorías:
1. muy bueno:
16-18 ptos.
2. bueno:
13-15 ptos.
3. regular:
10-12 ptos.
4. malo:
06-09 ptos.

Para determinar el impacto se realizó un cálculo a través de la puntuación de los parámetros establecidos previamente (Tabla 1). La determinación de los criterios de puntuación para calcular el impacto, se realizó a través de la consideración de las recomendaciones vigentes sobre lactancia materna (VALDÉS et al., 1994). En relación a la asistencia a controles, cumplimiento de acuerdos tomados y percepción de resolución de inquietudes de la madre, los criterios fueron establecidos por los investigadores tomando en consideración las experiencias en la Consulta de Apoyo. El puntaje total obtenido fue la suma del promedio de puntos obtenidos en cada respuesta por la muestra en estudio. Cabe recordar que el instrumento fue validado por expertos en el área. 
Tabla 1 - Dimensiones consideradas en la variable impacto

\begin{tabular}{|c|c|c|c|}
\hline VARUABLE & DIMENSION & SUBDIMENSION & INDICADOR \\
\hline \multirow[t]{6}{*}{ Irmpacto } & $\begin{array}{l}\text { Durxión de la } \\
\text { L.M.E. }\end{array}$ & $\begin{array}{c}6 \text { o más meses. } \\
3-5 \text { meses. } \\
0-3 \text { reses. }\end{array}$ & $\begin{array}{l}3 \\
2 \\
1\end{array}$ \\
\hline & $\begin{array}{c}\text { Edaden la que se } \\
\text { introdujo otros } \\
\text { alimentos }\end{array}$ & $\begin{array}{l}6 \text { o más meses. } \\
3-5 \text { reses. } \\
0-3 \text { reses. }\end{array}$ & $\begin{array}{l}3 \\
2 \\
1\end{array}$ \\
\hline & $\begin{array}{l}\text { Durción de la } \\
\text { lactancia total }\end{array}$ & $\begin{array}{l}8-12 \text { meses. } \\
47 \text { meses. } \\
0-3 \text { meses. }\end{array}$ & $\begin{array}{l}3 \\
2 \\
1\end{array}$ \\
\hline & $\begin{array}{c}\text { Asistencia a } \\
\text { controles }\end{array}$ & $\begin{array}{c}\text { Más de } 3 . \\
1-3 . \\
\text { Ninguno. }\end{array}$ & $\begin{array}{l}3 \\
2 \\
1\end{array}$ \\
\hline & $\begin{array}{l}\text { Curnple los } \\
\text { acuerdos } \\
\text { torrados }\end{array}$ & $\begin{array}{c}\text { Gereralmente. } \\
\text { Pancialmente. } \\
\text { No. }\end{array}$ & $\begin{array}{l}3 \\
2 \\
1\end{array}$ \\
\hline & $\begin{array}{l}\text { La madre percibe } \\
\text { que sus } \\
\text { incuietudes } \\
\text { fueron resueltas }\end{array}$ & $\begin{array}{c}\mathrm{Si} \\
\text { Parcialmente } \\
\text { No. }\end{array}$ & $\begin{array}{l}3 \\
2 \\
1\end{array}$ \\
\hline
\end{tabular}

\section{RESULTADOS}

La población estudiada corresponde a mujeres en un 60\% adultas jóvenes (20-30 años), todas con pareja estable, y en el $63.3 \%$ de los casos primíparas. E1 $60 \%$ de ellas tiene educación superior, y un $80 \%$ se desempeña como empleadas de oficina. La gran mayoría pertenece a un nivel socio económico medio.

En relación a los factores predisponentes, facilitadores y reforzadores estudiados, el resultado más significativo fue con respecto al apoyo recibido por las madres de alguna persona cercana para continuar amamantando, una vez reiniciada su actividad laboral. Según el análisis de los resultados obtenidos a través del método de sobre vida de Kaplan-Meier, existe una diferencia significativa en las probabilidades de seguir lactando según el apoyo recibido. Esto se puede apreciar en el primer gráfico, donde todas las madres comienzan con un $100 \%$ de probabilidad de amamantar la cual se mantiene hasta el $4^{\circ}$ mes. Luego se produce una diferencia entre las madres que tuvieron el apoyo de alguna persona cercana, que las alentara a continuar la lactancia después de reincorporarse a su trabajo. Estas madres se mantienen con una probabilidad de un $100 \%$ de seguir amamantando hasta el sexto mes aproximadamente. En cambio en las madres que no se sintieron apoyadas, al cuarto mes disminuye a un $80 \%$ la probabilidad de seguir lactando, la que continua decreciendo a través del transcurso de los meses, en relación al otro grupo de madres, en el cual el descenso se produce en forma más paulatina y menos marcada. De hecho el gráfico muestra una disminución al 50\% de probabilidad de amamantar en el sexto mes en el grupo de madres sin apoyo.

Se ha descrito, que los apoyos situacionales, tanto en la familia como en el trabajo, constituyen el pilar fundamental y reforzador para la mantención de la lactancia materna. Así mismo el compromiso social a través del apoyo del empleador, y del equipo de salud, significan un incentivo continuo para la madre (SCHELLHORN \& VALDÉS, 1995).

Gráfico 1 - Curvas de análisis por el método de Kaplan-Meier de la probabilidad de amamantar según el apoyo de alguna persona cercana recibido por las madres atendidas en la "consulta de apoyo a la madre que trabaja" del C.E.D.I.U.C entre marzo y septiembre de 1995

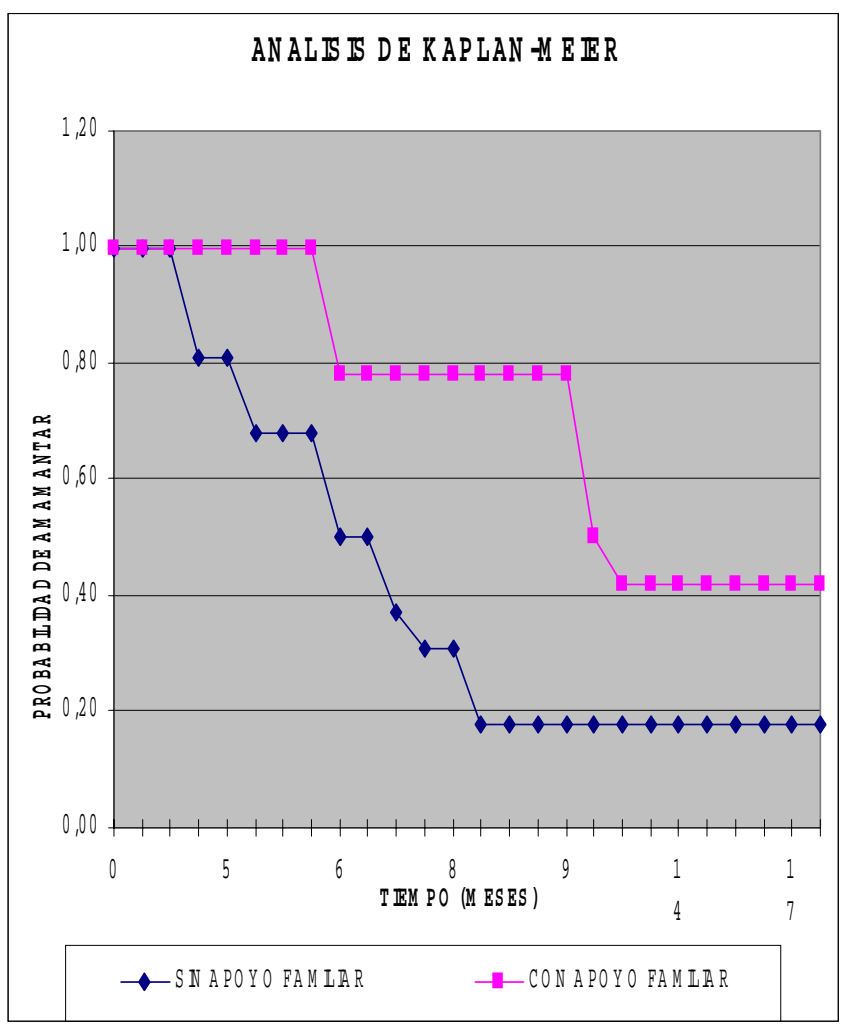

Un $46.7 \%$ de las madres logró mantener L.M.E. hasta los seis meses, y un $23.3 \%$ lo hizo hasta los cinco meses. Sólo $16.6 \%$ alcanzó solo tres meses de L.M.E., tal como se muestra en el Gráfico 2.

Si comparamos los resultados de este estudio con los porcentajes a nivel nacional, se aprecia un incremento importante en la prolongación de la L.M.E., ya que el porcentaje de niños con L.M.E. a los 4 meses en el grupo de estudio alcanza un $80 \%$ en relación a un $44.1 \%$ en el país. 
Gráfico 2 - Tiempo de L.M.E. de las madres que asisten a la "consulta de apoyo a la madre que trabaja" del C.E.D.I.U.C entre marzo y septiembre 1995

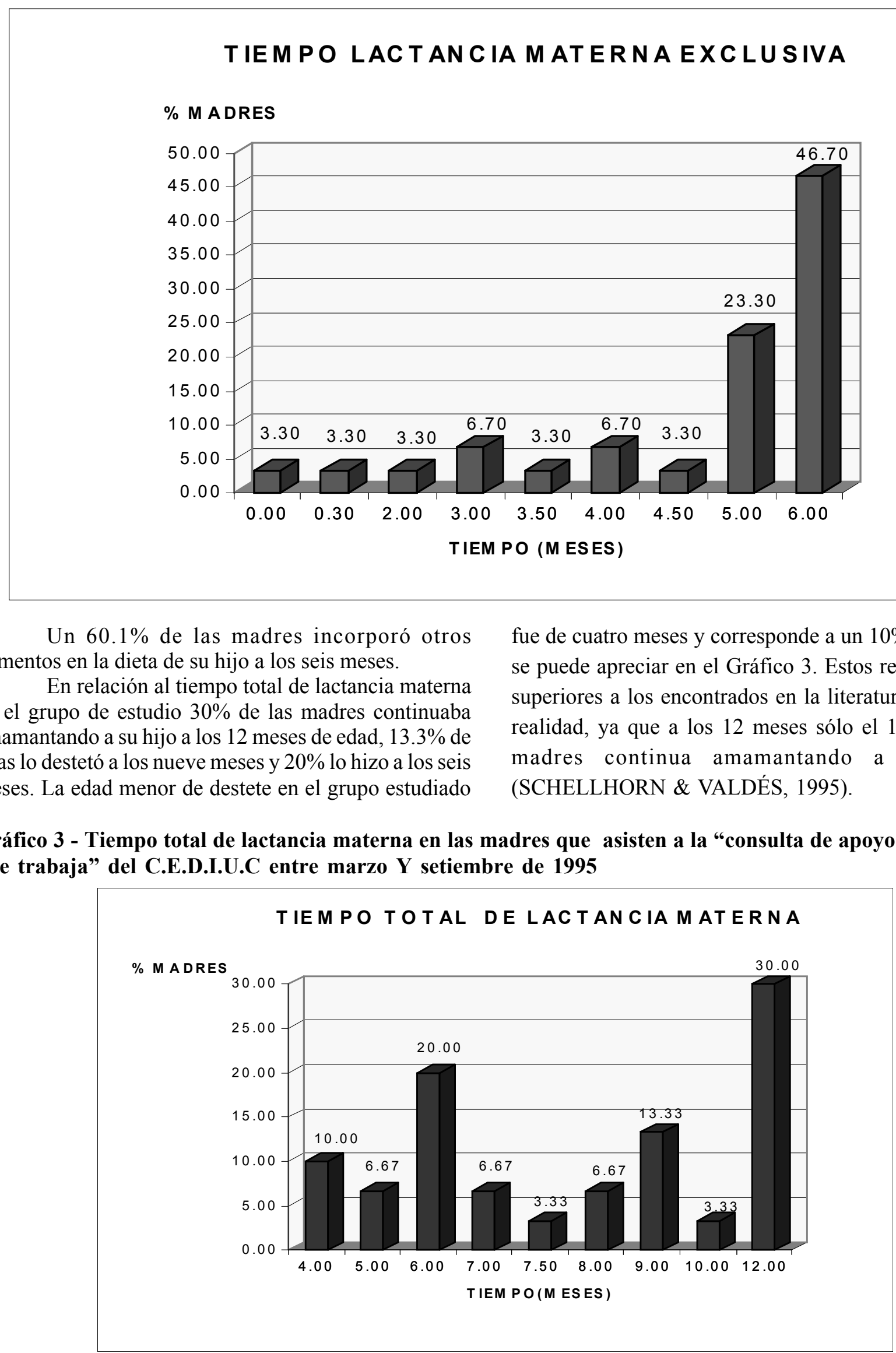


De las madres que abandonaron la lactancia antes de los doce meses, la principal causa de abandono del amamantamiento mencionado por ellas aparece en la Tabla $\mathrm{N}^{\circ} 2$, destacándose la escasa producción de leche $(33.33 \%)$ y el estrés en el trabajo (28.57\%). Este resultado tiene relación con que el tipo de trabajo en sí que realiza la madre, incide directamente en la mantención de su lactancia. El estrés y la tensión a la que se encuentra sometida por una parte, así como el horario de la jornada laboral, por ejemplo, condicionará el tiempo que debe estar separada de su hijo, y por lo tanto el número de mamadas que deberá reemplazar por extracciones de leche. Es así que mientras mayor sea el tiempo que ella debe estar fuera del hogar, disminuirá el número de mamadas en 24 horas, con la consecuente disminución del estímulo para la producción de leche. La disponibilidad de tiempo y un lugar físico en su trabajo para extraerse leche se relaciona directamente con el número de veces que la madre podrá vaciar sus mamas para mantener de ese modo su producción láctea. Al mismo tiempo la disponibilidad de sala cuna y de tiempo para amamantar al niño facilita la mantención de la lactancia (WABA \& UNICEF, 1993).

Tabla 2 - La principal causa del abandono de la lactancia materna mencionada por las madres que asisten a la "consulta de apoyo a la madre que trabaja"del C.E.D.I.U.C entre marzo Y septiembre de 1995

\begin{tabular}{l|c|c}
\hline \multicolumn{1}{c|}{ Motivo de abandono } & N $^{\circ}$ de madres & $\mathbf{\%}$ \\
\hline escasa producción de leche & 7 & 33.33 \\
estrés & 6 & 28.57 \\
problema de la madre & 5 & 23.80 \\
rechazo del niño & 2 & 9.52 \\
falta de tiempo & 1 & 4.76 \\
\hline TOTAL & 21 & $100 \%$ \\
\hline
\end{tabular}

En relación a la consulta de apoyo $73.3 \%$ de las madres se sintió muy acogida por la enfermera que la atendió el $20 \%$ acogida y el $6,7 \%$ poco acogida. Las madres resolvieron sus inquietudes en un $70 \%$ en relación a los aspectos legales. En un $86.7 \%$ en relación a la angustia por la separación de su hijo y en un $96.7 \%$ con respecto a la extracción y manejo de la leche materna.

En relación al número de controles de la consulta a los que asistieron las madres un $53.3 \%$ no asistió a control, un $36.7 \%$ fue a tres controles o menos y un $10 \%$ fue a más de tres controles.

Con respecto a la adherencia a los acuerdos tomados en la consulta un $43.3 \%$ de las madres cumplió dichos acuerdos, un 30\% lo hizo parcialmente, un $13.3 \%$ no lo hizo y un $13.4 \%$ no lo recuerda. El $40 \%$ de las madres opinó que la consulta es buena, 23.3\% la calificó excelente, $20 \%$ muy buena, $13.3 \%$ necesaria y un $3.4 \%$ restante no opinó. En relación a la utilidad de la consulta,
96.7\% de las madres encuestadas considera que la consulta es útil y el $100 \%$ de ellas la recomendaría a otras madres.

En relación al cumplimiento de los beneficios legales que le corresponden a las madres en el grupo estudiado, $73.4 \%$ recibe tiempo para alimentar a sus hijos, $73.3 \%$ recibe tiempo para extraerse leche, $76.7 \%$ cuenta con alguna persona que cuide a su hijo y $56.3 \%$ tiene derecho a sala cuna. Todas las madres del estudio estuvieron los tres primeros meses de vida del niño junto a él.

En relación al impacto de la consulta de apoyo a la madre que trabaja se concluye que un $77.9 \%$ alcanzó un buen impacto.

Esta experiencia vivida en el C.E.D.I.U.C. con la Consulta de Apoyo a la madre que trabaja, es comparable con grupos de apoyo de la lactancia materna, creados por La Liga de La Lactancia Materna en Guatemala, Honduras y otros países. Ahí, se capacitaron madres como monitoras, encargadas de dirigir grupos de mujeres (embarazadas y madres que amamantan) dentro de una comunidad, las que se reúnen con el fin de recibir información, reflexionar y darse apoyo en lo que se refiere a la lactancia. Estos grupos han tenido un impacto en la calidad y prolongación de la lactancia en sus comunidades (LIGA de la Lactancia Materna de Guatemala, 1996).

\section{CONCLUSIONES Y SUGERENCIAS}

El apoyo de una persona cercana es el factor reforzador más significativo para la prolongación de la lactancia en las madres estudiadas. La consulta enfocada hacia las madres que trabajan realizada en el CEDIUC es una instancia de apoyo que tiene un «buen» impacto en la prolongación de la lactancia en las madres estudiadas. La enfermera matrona es uno de los profesionales del área de la salud que cumple un rol muy importante en la protección y fomento de la lactancia materna.

$\mathrm{Si}$ todas las madres consideran útil y recomendarían esta consulta es una necesidad real que debe ser optimada y promovida.

Cabe destacar que según la experiencia en la Consulta de Apoyo a la madre que trabaja, podría bastar un promedio de tres controles para obtener un impacto positivo en la prolongación de la lactancia materna. Así la enfermera tiene a lo menos tres oportunidades para intervenir, actuar y evaluar, como agente facilitador y de buena acogida para la madre en el proceso de mantención de la lactancia 
Se sugiere realizar estudios posteriores multivariados en los cuales se establezcan las diferencias comparativas entre un grupo de madres que asiste a la consulta y otro que no asiste. Mejorar la calidad de los registros utilizados en la consulta. Realizar un seguimiento formal (control) de las madres que asisten a la consulta y, evaluar periódicamente los resultados obtenidos por la consulta.

\section{BREAST FEEDING: IMPACT OF THE SUPPORT CONSULTATION TO THE WORKING MOTHER}

This descriptive, exploratory, retrospective and transversal investigation tries to answer the following questionnaire: What is the impact that the Support Consultation to the working mother-Diagnosis Center of the Pontifical Catholic University of Chile- has on breast-feeding prolongation? The population that has been studied is formed by 82 mothers attended in the Support Consultation during March '95/ September '95 period and from which a number of 30 people were taken as a sample. The impact of this consultation was evaluated with an instrument employed during an interview to each mother. Data were statistically analyzed with EPIINFO, the Kaplan-Meier survival method and the Mantel-Haenszel test to compare curves of survival. During data analysis authors found that mothers are mainly young adults, stable couples, first-time mothers, with technical and/or professional educational level, chiefly working as office clerks with full-time jobs and having a significant difference between existing minimum salary and the maximum one they earn. The results of this investigation lead us to the conclusion that mothers obtained an exclusive breast-feeding and ideal weaning age.The power of resolution of the Consultation -according to mothers- was satisfactory. The support given to the mother after her reincorporation to work is the most significant intensifier factor in relation with the increase in the probability of keeping on breast-feeding. In conclusion, the Consultation has good impact.

KEY WORDS: breast feeding, working mother, intensifier factors, breast feeding prolongation, weaning

\section{ANEXO 1}

INSTRUMENTO DE EVALUACIÓN DE LA CONSULTA DE APOYO A LA MADRE QUE TRABAJA

$\mathbf{N}^{\circ}$ FICHA:

FECHA:

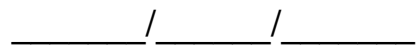

NOMBRE DEL NIÑO:

\section{Antecedentes maternos}

1. Nombre:

2. Edad:

3. Estado civil:
a) Soltera
b) Casada
c) Conviviente
d) Separada
e) Otro

4. Número de hijos:

5. Escolaridad:
a) Básica
b) Media
c) Superior

6. Último año cursado:

7. Actividad que desempeña:

8. Horario de trabajo:

9. Ingreso familiar:

\section{Factores predisponentes, facilitadores y reforzadores}

10.¿Tenía Ud. algún mito en relación a la lactancia materna, antes de acudir a la consulta?

NI $\quad(\quad)$ ¿Cuáles?


11.¿Tenía Ud. alguna creencia en relación a la lactancia materna, antes de acudir a la consulta?

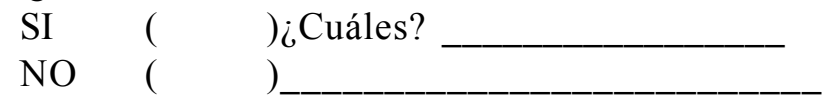

12.Si tiene más hijos, ¿Hasta qué edad (meses) amamantó a su(s) otro(s) hijo(s)?

Primer hijo

Segundo hijo

Tercer hijo

Cuarto hijo

Quinto hijo

13.¿Cómo fue(fueron) su(s) experiencia(s) anterior(es)?

$\begin{array}{lllll}\text { Primer hijo } & \text { Muy Buena } & \text { Buena } & \text { Regular } & \text { Mala } \\ \text { Segundo hijo } & \text { Muy Buena } & \text { Buena } & \text { Regular } & \text { Mala } \\ \text { Tercer hijo } & \text { Muy Buena } & \text { Buena } & \text { Regular } & \text { Mala } \\ \text { Quinto hijo } & \text { Muy Buena } & \text { Buena } & \text { Regular } & \text { Mala }\end{array}$

14.En su trabajo:

a) ¿Se le otorga a Ud. tiempo para alimentar a su hijo(a)?

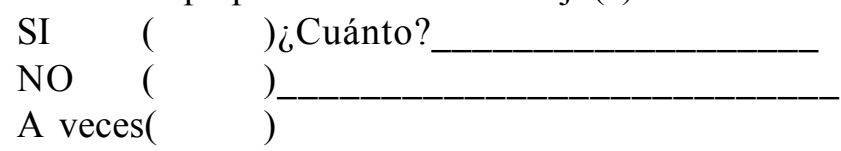

b) ¿Se le otorga a Ud. tiempo para extraerse leche?

SI $\quad(\quad)$ ¿Cuánto?
NO ()$_{-}$

c) ¿Se le otorga la posibilidad de llevar a su hijo a la sala cuna?

$\begin{array}{lll}\text { SI } & (\quad) \\ \text { NO } & (\quad)\end{array}$

15.¿Cuenta Ud. con alguna persona que cuide a su hijo mientras trabaja?

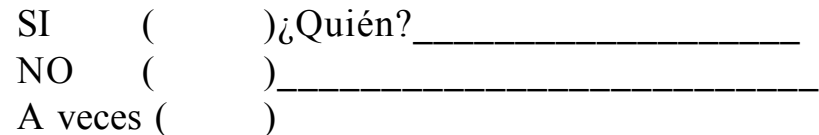

16.En relación a su lactancia : ¿Contó con el apoyo de alguna persona que la alentara a continuar después de la reincorporación de Ud. al trabajo?

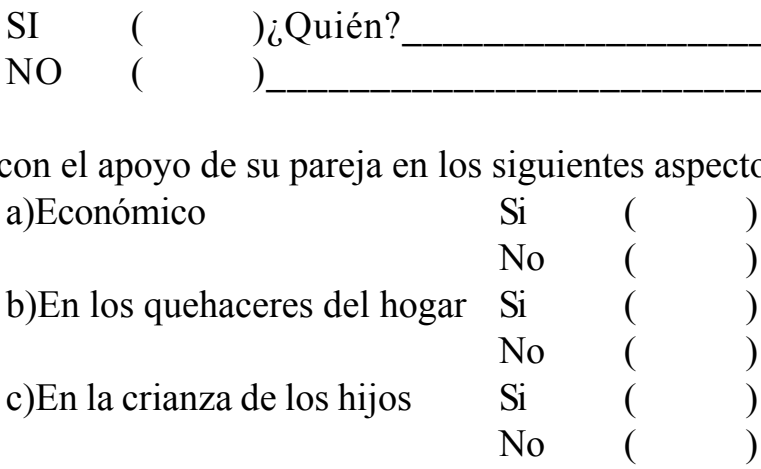

18.En relación a la enfermera-matrona que la orientó en la consulta de apoyo, Ud. se sintió:
a) Muy acogida ( )
b) Acogida $\quad(\quad)$
c) Poco acogida ( )
d) Nada acogida $(\quad$ ) 


\section{Tiempo de duración de la lactancia materna}

19.¿Hasta qué edad (último mes) amamantó Ud. en forma exclusiva a su hijo?

(mes)

20.¿A qué edad (el primer mes) comenzó Ud. a incorporan otros alimentos en la dieta de su hijo? (mes)

21.¿A qué edad (último mes) destetó Ud. a su hijo?

(mes)

22.¿Cuál fue la razón principal por la que abandonó la lactancia materna?

\section{Poder de resolución de la consulta}

23. ¿Siente Ud. que la consulta de apoyo, le sirvió para resolver las inquietudes que tenía en ese momento con respecto a :

a) Los beneficios legales que apoyan la lactancia materna.

$\begin{array}{ll}\mathrm{Si} & ( \\ \mathrm{No} & (\quad) \\ \text { Parcialmente } & (\end{array}$

b) Las preocupaciones e incertidumbres frente a la separación de su hijo por su reincorporación al trabajo.

$\begin{array}{ll}\mathrm{Si} & ( \\ \text { No } & (\quad) \\ \text { Parcialmente } & (\end{array}$

c)Extracción, conservación,almacenamiento y transporte de la leche materna.

$\begin{array}{ll}\mathrm{Si} & ( \\ \text { No } & ( \\ \text { Parcialmente } & (\quad)\end{array}$

d) Otras. ¿Cuáles?

$\begin{array}{ll}\mathrm{Si} & (\quad) \\ \text { No } & (\quad) \\ \text { Parcialmente } & (\end{array}$

\section{Adherencia a los acuerdos tomados en la consulta}

24.¿Cumplió Ud. con los acuerdos tomados en la consulta?

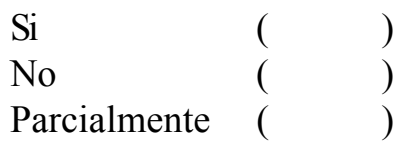

\section{Número de controles a los que asistió}

25.¿ A cuántos controles asistió Ud. posteriormente en la clínica de lactancia?

$\mathrm{N}^{\mathrm{o}}$ de controles

26. Revisar en la ficha el número de controles a los que asistió la madre.

$\mathrm{N}^{\mathrm{o}}$ de controles

\section{En general :}

a)¿Qué opina Ud. de la consulta de apoyo? 
b) ¿Le fue de utilidad asistir a la consulta de apoyo?

$$
\begin{aligned}
& \text { Sí } \\
& \text { No sé ¿Por qué? }
\end{aligned}
$$

c) ¿Recomendaría Ud. esta consulta a otras madres?

$$
\begin{aligned}
& \text { Sí } \\
& \text { No } \\
& \text { No sé ¿Por qué? }
\end{aligned}
$$

\section{REFERÊNCIAS BIBLIOGRÁFICAS}

01. CASADO, M.E.; CORONEL F.; VIDAL, L. Lactancia materna. Santiago: Editorial Mediterráneo, $1992.139 \mathrm{p}$.

02. COURT, I. La situación laboral de la mujer en Chile. Santiago, 1996. 204 p. Tesis (Periodista) Escuela de Periodismo, Universidad Gabriela Mistral.

03. GALAZ, L.M.; LIVESEY, A.; NAVARRO, S. Factores que influyen en la lactancia que reciben los hijos de primíparas precoces. Santiago, 1994. Tesis (Enfermera-matrona) Escuela de Enfermería, Pontificia Universidad Católica de Chile.

04. GREEN, L.W. et al. Health education planning a diagnostic approach. USA, Palo Alto (CA): Mayfield Publishing, 1980.

05. GUZMAN, P. et al. Modelos teóricos en educación para la salud. PAS, v. 7, n. 2, p. 712, 1990.
06. LIGA de la Lactancia Materna de Guatemala (1996). ¿Cómo organizar grupos de apoyo de lactancia materna?. In: NAVIN, A. (Eds.) Manual para monitoras en lactancia materna. San Diego, CA: WellStart International, 1996. p. 46-48. (Programa Educativo del manejo de la Lactancia).

07. MINSAL. Ministerio de Salud. División de Programas de Salud. Comisión Nacional de Lactancia Materna. Lactancia materna: derecho de la mujer trabajadora. Santiago: Chile, 1993. p. 7-20.

08. SCHELLHORN, H.C.; VALDÉS, L.V. Lactancia materna: contenidos técnicos para profesionales de la salud. Santiago: Comisión Nacional de Lactancia Materna. M.S./UNICEF, 1995. 230p.

09. VALDÉS, V.; PÉREZ, A.; LABBOK, M. Lactancia para la madre y el niño. Santiago: Editorial Merditerráneo, 1994. $142 \mathrm{p}$.

10. WABA; UNICEF. Mujer, trabajo y lactancia materna. In: Programa educativo del manejo de la lactancia. Folleto de Acción para la iniciativa lugares de trabajos amigos de la madre. Penang. Malaysia: W.A.B.A Secretariat, 1993. 6p. 